\title{
openheart Colchicine for symptomatic coronary artery disease after percutaneous coronary intervention
}

\author{
Kah Long Aw (1) , ${ }^{1,2}$ Amanda Koh, ${ }^{3}$ Han Lin Lee, ${ }^{4}$ Aurimas Kudzinskas, ${ }^{1}$ \\ Rodney De Palma ${ }^{2}$
}

\begin{abstract}
- Additional supplemental material is published online only. To view, please visit the journal online (http://dx.doi.org/10.
\end{abstract} 1136/openhrt-2021-001887).

To cite: Aw KL, Koh A, Lee HL, et al. Colchicine for symptomatic coronary artery disease after percutaneous coronary intervention. Open Heart 2022;9:e001887. doi:10.1136/ openhrt-2021-001887

KLA and AK contributed equally.

Received 12 0ctober 2021 Accepted 22 November 2021

A) Check for updates

C) Author(s) (or their employer(s)) 2022. Re-use permitted under CC BY-NC. No commercial re-use. See rights and permissions. Published by BMJ.

${ }^{1}$ Oxford University Hospitals NHS Trust, Oxford, UK

${ }^{2}$ Wycombe Hospital Department of Cardiology, Buckinghamshire Healthcare NHS Trust, High

Wycombe, UK

${ }^{3}$ Imperial College Healthcare

NHS Trust, London, UK

${ }^{4}$ Royal Berkshire NHS

Foundation Trust, Reading, UK

Correspondence to

Dr Kah Long Aw; awkahlong@ gmail.com

\section{ABSTRACT}

Background Percutaneous coronary intervention (PCI), the preferred coronary reperfusion strategy, induces endothelial trauma which may mount an inflammatory response. This has been shown to increase the likelihood of further major adverse cardiovascular events (MACE). Colchicine, a cheap and widely used anti-inflammatory has shown promise in improving cardiovascular outcomes. We aimed to perform a systematic review and metaanalysis to study the effects of colchicine in patients with symptomatic coronary artery disease (CAD) who have undergone $\mathrm{PCl}$.

Method We systematically reviewed and meta-analysed 7 randomised controlled trials including a total of 6660 patients (colchicine group: 3347, control group: 3313; mean age $=60.9 \pm 10$ ). Six studies included participants who had a $\leq 13.5$-day history of acute coronary syndrome (ACS). One study included patients with both ACS and chronic coronary syndrome. The follow-up of studies ranged from 3 days to 22.6 months.

Results The use of colchicine in patients who underwent $\mathrm{PCl}$ significantly reduced MACE outcomes (risk ratio 0.73 (95\% Cl 0.61 to 0.87$) ; p=0.0003$ ) with minimal heterogeneity across the analysis $\left(\mathrm{I}^{2}=6 \%\right.$; $\mathrm{P}$ for Cochran $Q=0.38$ ). These results were driven mainly by the reduction in repeat vessel revascularisation, stroke and stent thrombosis. The number needed to treat to prevent one occurrence of MACE was 41.

Conclusion Colchicine significantly reduced the risk of MACE in patients with CAD who underwent PCI, mostly in the reduction of repeat vessel revascularisation, stroke and stent thrombosis. The efficacy of colchicine should be further studied by distinguishing its use alongside different stent types and dosing regimens.

PROSPERO registration number CRD42021245699.

\section{INTRODUCTION}

Current coronary artery disease (CAD) treatment is multifaceted, involving a combination of lifestyle modifications, drugs such as antihypertensive regimens, antithrombotic therapy, lipid-lowering therapy and if necessary, medical procedures such as percutaneous coronary intervention (PCI) and coronary artery bypass graft surgery. ${ }^{1}$ Despite these treatments, residual risk of cardiovascular

\section{Key questions}

What is already known about this subject?

- Verma et al, Xia et al and Samuel et al have shown a reduction in composite major cardiovascular adverse outcomes in patients with coronary artery disease (CAD) when low-dose colchicine is used alongside guideline-therapy consisting of pharmaceutical \pm interventional therapy. Furthermore, Khandkar et al, Masson et al and Katsanos et al showed a reduction specifically of stroke incidence in patients with CAD when treated with low-dose colchicine. However, new results from major primary trials investigating the benefits of colchicine in CAD have emerged recently.

What does this study add?

- Our study adds to the literature by quantifying the benefits of the anti-inflammatory effects of colchicine following percutaneous coronary intervention (PCl). Our study is novel in two ways: (1) we study the effects of colchicine only in patients who underwent both $\mathrm{PCl}$ and medical therapy and (2) we provide an updated systematic review and metaanalysis including a recently published major trial - the Colchicine in Patients with Acute Coronary Syndrome (COPS) trial.

How might this impact on clinical practice?

- Colchicine is a cheap and relatively low risk medication which may be beneficial $(27 \%$ risk reduction, number needed to treat=41) for patients undergoing $\mathrm{PCl}$ in reducing major cardiovascular events and disease morbidity. However, more studies need to be conducted to investigate the effects of colchicine in a periprocedural versus secondary prevention setting.

events during the first 365 days after a primary myocardial infarction (MI) remains at $22 \%,{ }^{2}$ suggesting that the current treatment regime can be further optimised.

The role of inflammation in all stages of pathogenesis of CAD has been long established. $^{3}$ Higher levels of inflammatory markers are associated with the occurrence of coronary thrombosis and acute coronary 
syndromes (ACS). ${ }^{4}$ Endothelial damage during PCI with stent implantation induces a further inflammatory response. ${ }^{5}$ The periprocedural inflammatory status of patients undergoing PCI has been shown to independently affect the prognosis of subsequent cardiovascular events. ${ }^{67}$ Post-PCI, MI occurred in $7.5 \%$ of patients with persistent residual inflammatory risk, compared with $4.3 \%$ of patients with low residual inflammatory risk. ${ }^{8}$ Furthermore, studies have also shown an increased risk of restenosis, target vessel revascularisation (TVR) and death in patients with raised inflammatory markers. ${ }^{89}$ Thus, it has been hypothesised that reducing inflammation after an acute MI should improve patient outcomes.

Targeting inflammation is an emerging avenue for novel therapeutic agents in an ACS setting. The beneficial role of anti-inflammatories in CAD was emphasised following the publication of the Canakinumab Antiinflammatory Thrombosis Outcome Study, which demonstrated a reduction of secondary cardiovascular events in patients with a raised high-sensitivity $\mathrm{C}$ reactive protein by inhibition of the NLRP3 inflammasome-dependent pathway via interleukin- $1 \beta^{3}$ pathway, without affecting lipid levels. ${ }^{10}$ Colchicine, a low-cost anti-inflammatory traditionally used in gout, has garnered new research interest as a potential candidate in cardiovascular disease prevention. Recent randomised controlled trials (RCTs) have demonstrated beneficial effects of colchicine for secondary cardiovascular disease prevention in patients with CAD. ${ }^{11-20}$ The early administration of colchicine as an adjunct to PCI for secondary prevention of cardiovascular events, however, is still uncertain. Our meta-analysis aimed to pool evidence by including RCTs to assess the efficacy of colchicine when used as an adjunct to PCI for the prevention of major adverse cardiovascular events (MACE).

\section{METHODS}

\section{Search strategy and selection criteria}

This systematic review and meta-analysis were conducted as per Preferred Reporting Items for Systematic Reviews and Meta-Analyses (PRISMA) guidelines and using the PICO tool $(p=$ patients with symptomatic CAD who underwent PCI; I=colchicine in addition to conventional guideline therapy; $\mathrm{C}=$ placebo in addition to conventional guideline therapy; $\mathrm{O}=$ major adverse cardiovascular events). A structured search was performed on EMBASE, MEDLINE and Cochrane Library for articles published from inception up to February 2021. Medical subject heading (MESH) terms and keywords were used to search for articles related to colchicine, acute coronary syndrome, acute coronary disease and percutaneous coronary intervention. Further details on the database and search terms used are shown in online supplementary material. After removal of duplicate articles, two reviewers (KLA and AKo) independently screened the articles using a two-step approach. First, abstracts and titles were screened for eligibility. The reviewers then screened the full-text articles. References of articles pertinent to the research question were screened for suitability (backward snowballing). The screening process is outlined in the PRISMA Flow Diagram (online supplementary material figure 1).

\section{Inclusion and exclusion criteria}

Our inclusion criteria were as follows; (1) studies which compare the efficacy of colchicine compared with placebo or no colchicine, in patients who underwent PCI, with reporting of MACE, (2) patients treated as per local guidelines for CAD, (3) study must be an RCT and (4) studies must be in English language.

\section{Data collection and risk of bias assessments}

Authors KLA and HLL extracted data systematically from the RCTs and used a standardised Microsoft Excel spreadsheet to record study design, population, size in colchicine arm (treatment) versus control arm (placebo or no treatment), age, sex, hypertension, diabetes mellitus, smoking history, PCI, antiplatelet therapy, statin therapy, time of colchicine initiation, colchicine dose, median follow-up, primary outcome and secondary outcome (table 1).

Each included full-text study was appraised using the Cochrane Risk Assessment Tool by authors KLA and AKu. The Cochrane Risk Assessment Tool Analysis and Overview analysis of Cochrane Risk Assessment can be found in online supplementary figures 2 and 3 .

\section{Outcomes}

Primary outcome measures were the MACE including in-stent restenosis (ISR), repeat vessel revascularisation, stent thrombosis, stroke, resuscitated cardiac arrest and all cause death. Contrary to the outcomes registered on International Prospective Register of Systematic Reviews, we did not include MI as part of MACE because this outcome was not reported in the included studies.

Secondary outcome measures include ISR, repeat vessel revascularisation, stent thrombosis, stroke and allcause death.

\section{Statistical analysis}

The Mantel-Haenszel random effects model ${ }^{21}$ was used to calculate the pooled relative risk (RR) and their corresponding $95 \%$ CIs of stroke incidence and safety outcomes of the RCTs included in this study. Heterogeneity was assessed using the $\mathrm{I}^{2}$ and Cochran $Q$ statistics. Number needed to treat (NNT) was calculated using the formula $\mathrm{NNT}=1 /[(1-\mathrm{RR}) \times$ outcome incidence in control groups $].{ }^{22}$ Funnel plots were assessed for publication bias by visual assessment. Using the 'metafor' package for R, the trim-and-fill method was applied to adjust for potential bias. ${ }^{23}$ All statistical analyses were conducted using the Cochrane Collaboration's Review Manager (RevMan V.5.3) Software Package (Copenhagen: Nordic Cochrane Centre, Cochrane Collaboration, 2014). 


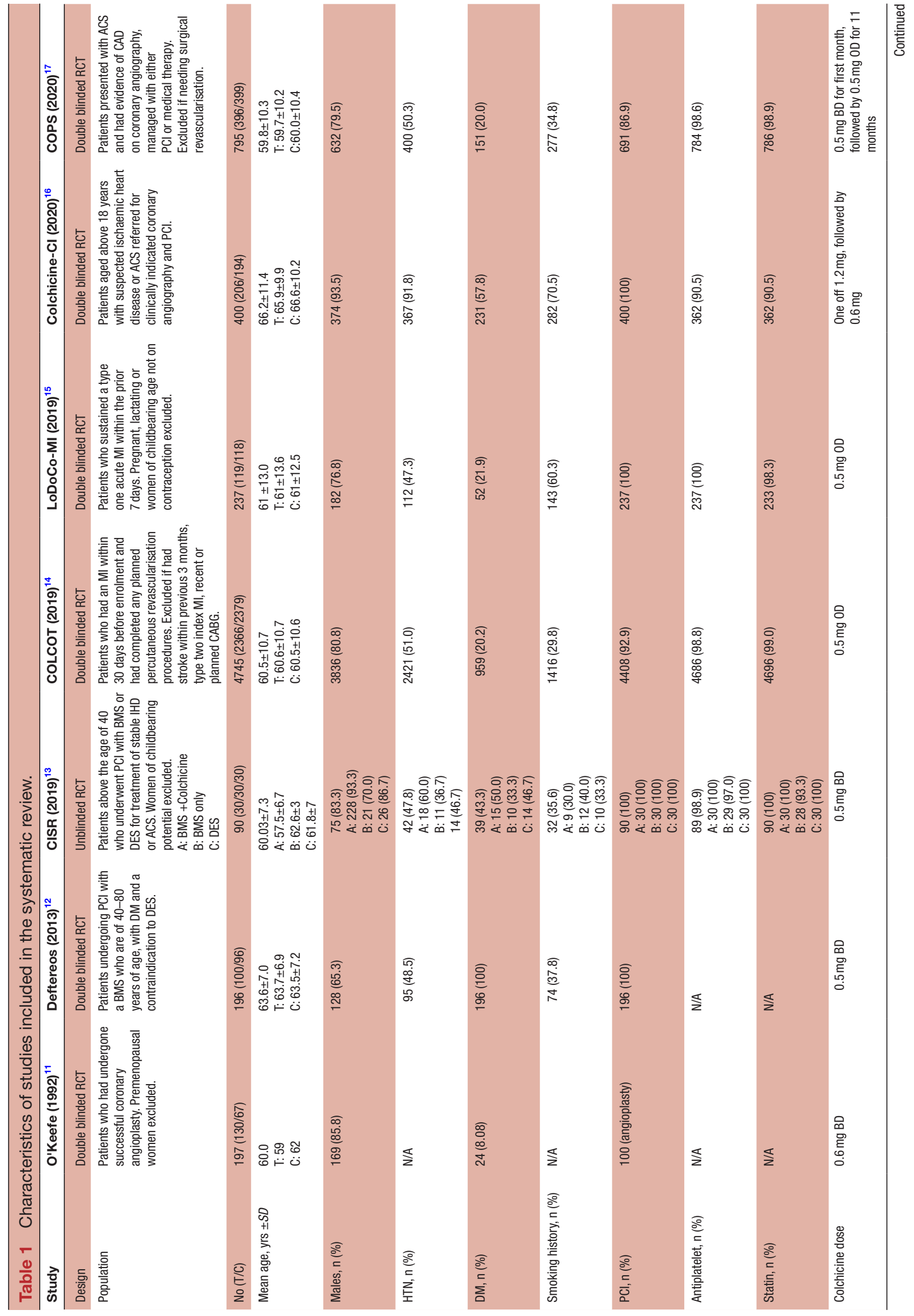




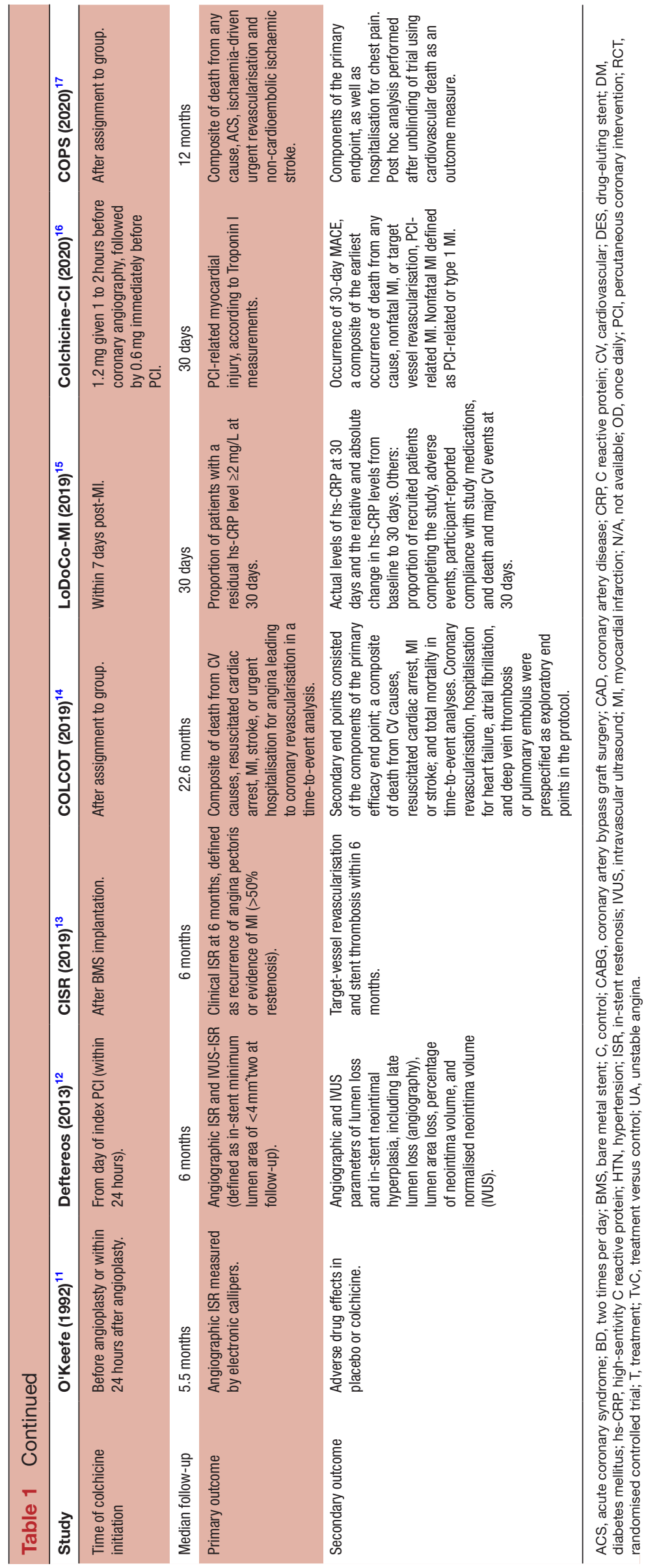


MACE

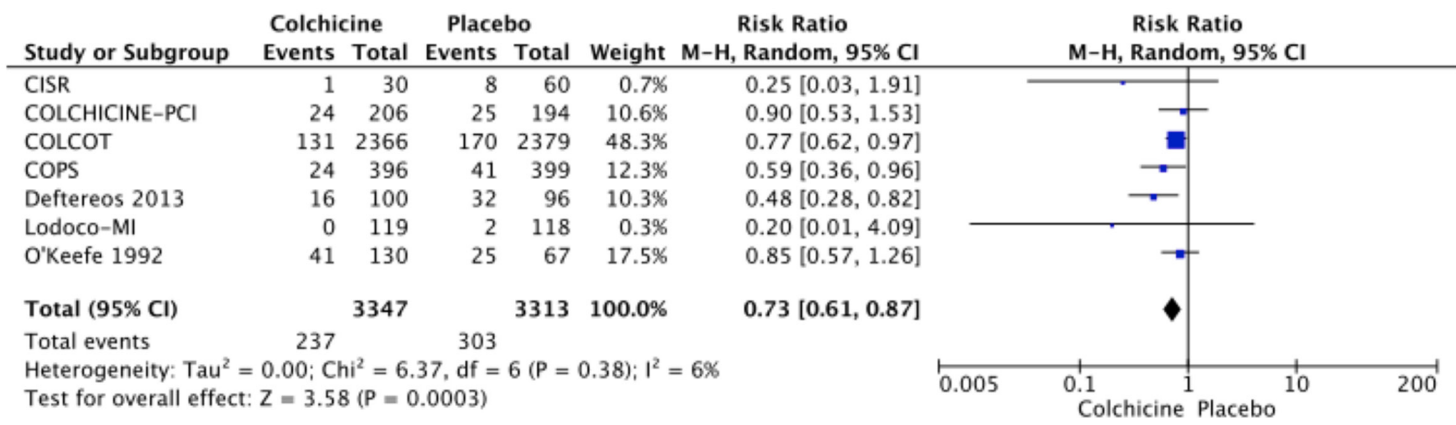

Figure 1 Primary outcome. Forest plot for MACE, showing pooled RRs of RCTs comparing patients who underwent PCl in the colchicine versus control group. RRs are random effects estimates calculated by Mantel-Haenszel (M-H) method. CISR, Colchicine Treatment for Prevention of in Stent Restenosis; COLCHICINE-PCI, Colchicine in Percutaneous Coronary Intervention; COLCOT, Colchicine Cardiovascular Outcomes Trial; COPS, Colchicine for Patients with Acute Coronary Syndrome; MACE, major adverse cardiovascular events; MI, myocardial infarction; PCI, percutaneous coronary intervention; $\mathrm{RCTs}$, randomised controlled trials; RRs, risk ratios.

\section{RESULTS}

A total of 121 abstracts and titles were screened, of which 105 were excluded as they did not study colchicine use in patients who underwent PCI. Of the 16 full-text articles assessed for eligibility, 7 were included in our systematic review and meta-analysis (figure 1). A list of excluded studies with reasons for exclusion can be found in online supplemental materials.

A total of 6660 participants (mean age: $60.9 \pm 10.6$, colchicine group $=3347$, control group $=3313$ ) were included in this study. Six studies recruited participants with a history of ACS of $\leq 13.5$ days. O'Keefe $e t a l$, recruited patients with both ACS and CCS. ${ }^{11}$ All participants from four studies, ${ }^{12} 13151692.9 \%$ from Tardif $e t a l^{14}$ and $86.9 \%$ from Tong et $a l^{17}$ had PCI for ACS. All participants in O'Keefe et al had elective balloon angioplasty. Colchicine was administered to patients after PCI in five studies, ${ }^{12-15} 17$ before PCI in one study, ${ }^{16}$ and either before or after balloon angioplasty in one study. ${ }^{11}$ The median follow-up ranged from 3 days to 22.6 months. The incidence of MACE in the colchicine group and control group were $237(7.08 \%)$ and $303(9.15 \%)$, respectively, and their individual components are summarised in table 2.

Risk of selection and detection bias were unclear in three studies which did not provide information on random sequence generation and outcome blinding (O'Keefe et $a l^{11}{ }^{11}$ Deftereos et $a l^{12}$ and Habib et al. ${ }^{13} \mathrm{~A}$ summary of the Cochrane Risk Assessment Tool can be found in online supplemental figures 2 and 3 .

\section{Primary outcome}

Quantitative analysis of pooled outcomes from seven RCTs showed that colchicine in patients who underwent PCI significantly reduced MACE outcomes (risk ratio 0.73 (95\% CI 0.61 to 0.87 ); $\mathrm{p}=0.0003$ ) with minimal heterogeneity across the analysis $\left(\mathrm{I}^{2}=6 \%\right.$; $\mathrm{P}$ for Cochran $\left.\mathrm{Q}=0.38\right)$ (figure 1).

\section{Secondary outcomes}

Three studies ${ }^{11-13}$ reported angiographically proven ISR. Meta-analysis showed no statistical significance in colchicine use for reduction of ISR for patients who underwent PCI (risk ratio 0.64 (95\% CI 0.36 to 1.15 ) ; $\mathrm{p}=0.14, \mathrm{I}^{2}=58 \%$; $\mathrm{P}$ for Cochran $\mathrm{Q}=0.09$ ) (figure 2).

Meta-analysis of four studies ${ }^{13} 141617$ showed a significant reduction in repeat vessel revascularisation when colchicine was used for patients who underwent PCI (risk ratio 0.47 (95\% CI 0.31 to 0.72$) ; \mathrm{p}=0.0004, \mathrm{I}^{2}=0 \%$; $\mathrm{P}$ for Cochran $Q=0.58$ ) (figure 2).

Furthermore, there was also a significant reduction in stent thrombosis when colchicine was given to patients who underwent PCI (risk ratio 0.50 (95\% CI 0.25 to 0.98); $\mathrm{p}=0.05, \mathrm{I}^{2}=0 \%$; $\mathrm{P}$ for Cochran $\mathrm{Q}=0.95$ ) (figure 2).

Pooled outcomes of seven RCTs showed a significant risk reduction in stroke when colchicine was used for patients who underwent PCI (risk ratio 0.50 (95\% CI 0.31 to 0.81$) ; \mathrm{p}=0.005, \mathrm{I}^{2}=0 \%$; $\mathrm{P}$ for Cochran $\mathrm{Q}=0.48$ ) (figure 2).

There was no significant difference in all-cause mortality whether colchicine is used in patients who underwent PCI (risk ratio 1.12 (95\% CI 0.49 to 2.58); $\mathrm{p}=0.79, \mathrm{I}^{2}=23 \%$; P for Cochran $\mathrm{Q}=0.26$ ) (figure 2).

\section{Publication bias}

Visual inspection of the funnel plot (figure 3) reveals asymmetrical scatter with studies of larger effect sizes potentially being suppressed in the positive direction. This indicates significant risk of publication bias for our primary efficacy. The trim-and-fill identified two missing studies on the right side (online supplemental material). This model estimate risk ratio 0.7492 (95\% CI 0.5873 to $0.9110) ; \mathrm{p}<0.0001$ ) with minimal heterogeneity across the analysis $\left(\mathrm{I}^{2}=0 \%\right.$; P for Cochran $\left.\mathrm{Q}=0.9954\right)$. The findings remain statistically significant after adjusting for missing studies. 


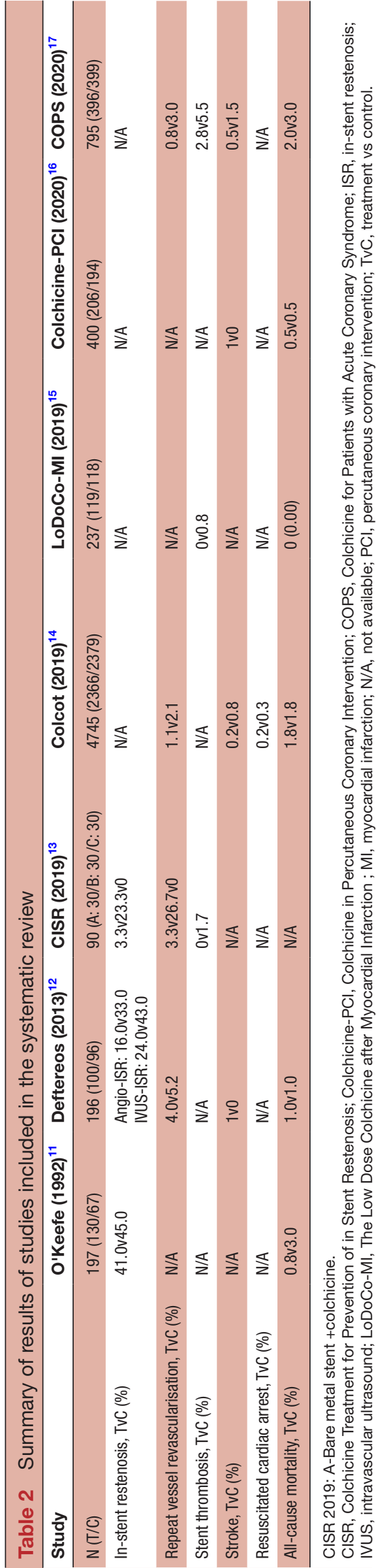

\section{DISCUSSION}

Our meta-analysis provides evidence that administration of colchicine early on, at the time of PCI reduces MACE (27\% risk reduction; NTT $=41)$. This risk reduction for the primary end point was mainly driven by lower rates of repeat vessel revascularisation, stroke and stent thrombosis.

The beneficial role of colchicine is likely explained by its wide-ranging effects on the inflammatory process. Colchicine concentrates in leukocytes and has a primary antimitotic effect against microtubule and spindle formation. ${ }^{24}$ It also induces downregulation of various inflammatory pathways further impacting neutrophil activation and recruitment, platelet aggregation and the expression of various cytokines and interleukins. ${ }^{24}$ From a clinical perspective, several studies demonstrated an increase of intracardiac production of the inflammasome-specific cytokines IL-1 $\beta$, IL-18 and downstream IL-6 in patients presenting with $\mathrm{ACS}^{25}$ and that acute colchicine administration was associated with a significant reduction in the transcoronary production of these cytokines. ${ }^{2627}$

While colchicine showed no significant effect on reducing in-stent stenosis in this study, it should be noted that there was some heterogeneity between the three RCTs studied for this outcome. O'Keefe $e t a l^{11}$ included patients who underwent balloon angioplasty with no stent implantation, the pathogenesis of which involves elastic recoil, vessel remodelling and neointimal proliferation. ${ }^{28}$ Colchicine possesses antiproliferation and anti-inflammatory properties, which may suggest that it is more suitable for PCI with stent implantation where the pathogenesis involves mainly neointimal proliferation and neoartherosclerosis. ${ }^{29}$ Furthermore, all patients in O'Keefe et al had a 6-month follow-up angiogram, suggesting that the findings included patients who potentially had asymptomatic in-stent stenosis. Meta-analysis of the other two papers alone (Habib et al and Deftereos et al) shows a significant reduction of $53 \%$ in ISR in the treatment group (risk ratio 0.46 (95\% CI 0.28 to 0.78 ); $\mathrm{p}=0.003$ ), with no heterogeneity seen across the studies $\left(\mathrm{I}^{2}=0 \%\right.$; $\mathrm{P}$ for Cochran $\left.\mathrm{Q}=0.09\right)$. Hence, another plausible explanation and hypothesis could be that colchicine reduces the severity of in-stent stenosis and hence reduces symptomatic stenosis. This is also supported by the fact that the rate of repeat vessel revascularisation is lower than ISR in this meta-analysis.

Limited data are available on the risks and impact of repeat vessel revascularisation. Since the advent of drugeluting stents (DES), the incidence of repeat vessel revascularisation has improved as compared with the use of bare-metal stents. ${ }^{30}$ However, despite optimal medical management and the use of DES, the 5-year cumulative incidence of repeat vessel revascularisation were demonstrated in two trials, to be as high as $20.33^{31}$ and $25.9 \% .^{32}$ Our study demonstrates that colchicine confers a risk reduction of $53 \%$ (risk ratio 0.47 (95\% CI 0.31 to 0.72 ); $\mathrm{p}=0.0004, \mathrm{I}^{2}=0 \%$; P for Cochran $\mathrm{Q}=0.58$ ) in repeat vessel 


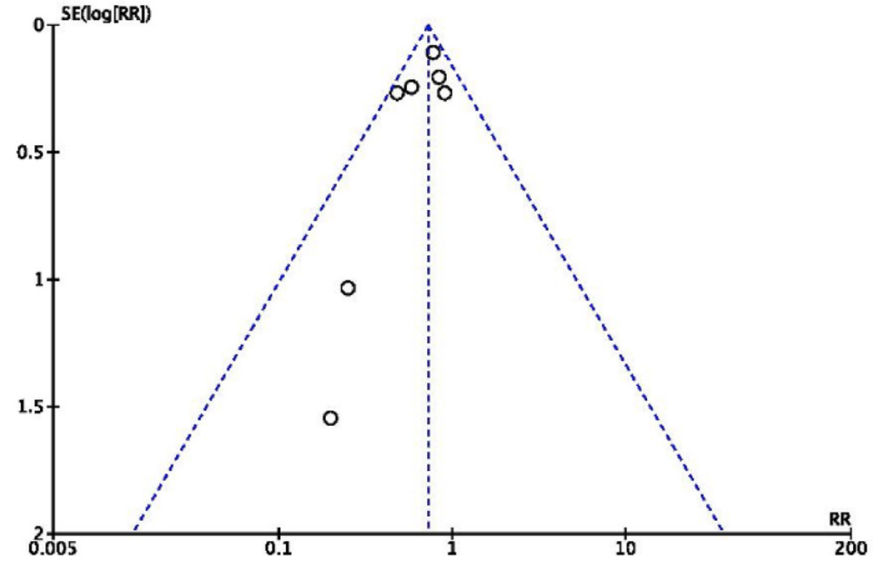

Figure 3 Funnel plot to assess publication bias. RR, risk ratios.

lesions whereas another study showed that both were performed equally. The anti-inflammatory and antiproliferative properties of colchicine likely benefit repeat intervention at both the site of index PCI and de novo lesions caused by ongoing atherosclerotic disease. More data are needed to establish if this beneficial effect is more pronounced in TVR or de novo lesions.

The reduction seen in stroke incidence is in line with previous studies. The risk of ischaemic stroke after a MI has been shown to be $2.7 \%$ at 2 years. ${ }^{31}{ }^{33}$ In the acute phase of MI, activated inflammasomes within myocardial fibroblasts mount an intense inflammatory response. ${ }^{34}$ For patients undergoing PCI, this is followed by periprocedural inflammation likely secondary to endothelial damage. ${ }^{5-7}$ This may contribute to the atherosclerotic plaque destabilisation and thromboembolism, causing cerebrovascular events. Colchicine's anti-inflammatory properties may have a role in the prevention of stroke caused by instability of native atherosclerotic plaques in patients who have undergone PCI.

There was also no significant change in all-cause mortality between patients given colchicine and the control group. In fact, there was a higher rate of total death in the colchicine group observed in the COPS trial. ${ }^{17} \mathrm{~A}$ focused meta-analysis which pooled data from the main trials on the topic showed a significant increase of non-CV death among colchicine-treated patients as compared with controls at an average follow-up of 25.1 months (OR 1.55, 95\% CI 1.10 to 2.17; $\mathrm{p}=0.010$ ). However, this was mostly attributed to the RCTs enrolling CCS patients and no specific cause of death responsible for this excess of deaths has been identified. ${ }^{24}$

Our paper has several limitations. First, O'Keefe et al had included patients who underwent balloon angioplasty with no stent implantation, which may be seen as heterogenous compared with other studies. The inflammatory response during balloon angioplasty may be similar to the one seen in stent placement which involves arterial puncture, administration of contrast agent, duration of fluoroscopy and endothelial injury. ${ }^{35}$ We hypothesised the cohort of patients undergoing balloon angioplasty will also benefit from the anti-inflammatory properties of colchicine. Second, Tong et al reported $86.9 \%$ of their study population had undergone PCI, the remaining patients had only been treated with medical management for ACS. We felt the number of patients treated with medical management was inadequate for us to ignore the benefit the study would provide to this review. The absolute number of patients who did not undergo PCI is relatively small and will unlikely affect results.

For colchicine to encounter clinical practice, further studies are required to fully assess its role in the treatment of ischaemic heart disease. There is promising potential in its use in a PCI setting, but further evaluation particularly in distinguishing between different stents (bare-metal vs drug-eluting), categorising patients based on MI type (ST elevation MI (STEMI) vs non-STEMI (NSTEMI)), as well as personalising colchicine use in terms of duration of treatment and dose would be needed. Trials such as the CLEAR SINERGY ${ }^{36}$ neutrophil substudy which examines clinical and genetic factors that determine heterogeneity in response to colchicine treatment may be a step in the right direction; suggesting that perhaps colchicine will be used in a selected population in the appropriate clinical setting.

\section{CONCLUSION}

Colchicine significantly reduces the risk of MACE in patients with symptomatic CAD who have undergone PCI. The largest benefit was seen in the reduction of ISR, stroke and stent thrombosis. Further clinical trials are required to evaluate the clinical benefits of colchicine use with different types of stents and alternative dosing regimens.

Acknowledgements We would like to thank Noureddine Kenssous for performing the literature search for us.

Contributors KLA: guarantor, idea conceptualisation, data collection, data interpretation and write up. AKo: idea conceptualisation, data collection, data interpretation and write up. HLL: data collection, data interpretation and write up. AKu: data collection, data interpretation and write up. RDP: supervision of project

Funding The authors have not declared a specific grant for this research from any funding agency in the public, commercial or not-for-profit sectors.

Competing interests None declared.

Patient consent for publication Not applicable.

Provenance and peer review Not commissioned; externally peer reviewed.

Data availability statement Data are available in a public, open access repository. This study will be available through open access.

Open access This is an open access article distributed in accordance with the Creative Commons Attribution Non Commercial (CC BY-NC 4.0) license, which permits others to distribute, remix, adapt, build upon this work non-commercially, and license their derivative works on different terms, provided the original work is properly cited, appropriate credit is given, any changes made indicated, and the use is non-commercial. See: http://creativecommons.org/licenses/by-nc/4.0/.

ORCID iD

Kah Long Aw http://orcid.org/0000-0003-4405-576X 


\section{REFERENCES}

1 Collet J-P, Thiele H, Barbato E, et al. 2020 ESC Guidelines for the management of acute coronary syndromes in patients presenting without persistent ST-segment elevation. Eur Heart $J$ 2021;42:1289-367.

2 Jernberg T, Hasvold P, Henriksson M, et al. Cardiovascular risk in post-myocardial infarction patients: nationwide real world data demonstrate the importance of a long-term perspective. Eur Heart $J$ 2015;36:1163-70.

3 Ross R. Atherosclerosis--an inflammatory disease. N Engl J Med 1999;340:115-26.

4 Libby P, Tabas I, Fredman G, et al. Inflammation and its resolution as determinants of acute coronary syndromes. Circ Res 2014;114:1867-79.

5 Cao D, Chiarito M, Mehran R. Treating inflammation prior to percutaneous coronary intervention: does the heart care? Circ Cardiovasc Interv 2020;13:e009127.

6 de Winter RJ, Heyde GS, Koch KT, et al. The prognostic value of preprocedural plasma $\mathrm{C}$-reactive protein in patients undergoing elective coronary angioplasty. Eur Heart J 2002;23:960-6.

7 Arroyo-Espliguero R, Avanzas P, Cosín-Sales J, et al. C-reactive protein elevation and disease activity in patients with coronary artery disease. Eur Heart J 2004;25:401-8.

8 Kalkman DN, Aquino M, Claessen BE, et al. Residual inflammatory risk and the impact on clinical outcomes in patients after percutaneous coronary interventions. Eur Heart J 2018;39:4101-8.

9 Toutouzas K, Colombo A, Stefanadis C. Inflammation and restenosis after percutaneous coronary interventions. Eur Heart $J$ 2004;25:1679-87.

10 Ridker PM, Everett BM, Thuren T, et al. Antiinflammatory therapy with canakinumab for atherosclerotic disease. N Engl J Med 2017;377:1119-31.

11 O'Keefe JH, McCallister BD, Bateman TM, et al. Ineffectiveness of colchicine for the prevention of restenosis after coronary angioplasty. J Am Coll Cardiol 1992;19:1597-600.

12 Deftereos S, Giannopoulos G, Raisakis K, et al. Colchicine treatment for the prevention of bare-metal stent restenosis in diabetic patients. J Am Coll Cardiol 2013;61:1679-85.

13 Habib M, Salama I, Agha HA, et al. Colchicine treatment for prevention of in stent restenosis (CISR trial). American J Emerg Crit Care Med 2019;2:001-5.

14 Tardif J-C, Kouz S, Waters DD, et al. Efficacy and safety of low-dose colchicine after myocardial infarction. $N$ Engl J Med 2019;381:2497-505.

15 Hennessy T, Soh L, Bowman M, et al. The low dose colchicine after myocardial infarction (LoDoCo-MI) study: a pilot randomized placebo controlled trial of colchicine following acute myocardial infarction. Am Heart J 2019;215:62-9.

16 Shah B, Pillinger $\mathrm{M}$, Zhong $\mathrm{H}$, et al. Effects of acute colchicine administration prior to percutaneous coronary intervention: COLCHICINE-PCI randomized trial. Circ Cardiovasc Interv 2020;13:e008717.

17 Tong DC, Quinn S, Nasis A, et al. Colchicine in patients with acute coronary syndrome: the Australian cops randomized clinical trial. Circulation 2020;142:1890-900.

18 Nidorf SM, Eikelboom JW, Budgeon CA, et al. Low-Dose colchicine for secondary prevention of cardiovascular disease. J Am Coll Cardiol 2013;61:404-10.
19 Nidorf SM, Fiolet ATL, Mosterd A, et al. Colchicine in patients with chronic coronary disease. N Engl J Med Overseas Ed 2020;383:1838-47.

20 Bouabdallaoui N, Tardif J-C, Waters DD, et al. Time-to-treatment initiation of colchicine and cardiovascular outcomes after myocardial infarction in the colchicine cardiovascular outcomes trial (COLCOT). Eur Heart J 2020;41:4092-9.

21 Mantel N, Haenszel W. Statistical aspects of the analysis of data from retrospective studies of disease. J Natl Cancer Inst 1959;22:719-48.

22 Mendes D, Alves C, Batel-Marques F. Number needed to treat (NNT) in clinical literature: an appraisal. BMC Med 2017;15:112.

23 Viechtbauer W. Conducting meta-analyses in $\mathrm{R}$ with the metafor package. J Stat Softw 2010;36:1-48.

24 Galli M, Princi G, Crea F, et al. Colchicine and risk of noncardiovascular death in patients with coronary artery disease: a pooled analysis underling possible safety concerns. Eur Heart $J$ Cardiovasc Pharmacother 2021;7:e18-19.

25 Pedicino D, Severino A, Ucci S, et al. Epicardial adipose tissue microbial colonization and inflammasome activation in acute coronary syndrome. Int J Cardiol 2017;236:95-9.

26 Martínez GJ, Robertson S, Barraclough J, et al. Colchicine acutely suppresses local cardiac production of inflammatory cytokines in patients with an acute coronary syndrome. $J$ Am Heart Assoc 2015;4:e002128.

27 Robertson S, Martínez GJ, Payet CA, et al. Colchicine therapy in acute coronary syndrome patients acts on caspase- 1 to suppress NLRP3 inflammasome monocyte activation. Clin Sci 2016;130:1237-46.

28 Teirstein PS, Massullo V, Jani S, et al. Catheter-based radiotherapy to inhibit restenosis after coronary stenting. $N$ Engl J Med 1997;336:1697-703.

29 Hoffmann R, Mintz GS, Dussaillant GR, et al. Patterns and mechanisms of in-stent restenosis. A serial intravascular ultrasound study. Circulation 1996;94:1247-54.

30 Alhejily W, Ohman E. Repeat revascularization after PCI. Circulation: Cardiovascular Interventions 2012;5:746-7.

31 Serruys PW, Onuma Y, Garg S, et al. 5-Year clinical outcomes of the ARTS II (arterial revascularization therapies study II) of the sirolimus-eluting stent in the treatment of patients with multivessel de novo coronary artery lesions. J Am Coll Cardiol 2010;55:1093-101.

32 Mohr FW, Morice M-C, Kappetein AP, et al. Coronary artery bypass graft surgery versus percutaneous coronary intervention in patients with three-vessel disease and left main coronary disease: 5-year follow-up of the randomised, clinical SYNTAX trial. Lancet 2013;381:629-38.

33 Yaghi S, Pilot M, Song $\mathrm{C}$, et al. Ischemic stroke risk after acute coronary syndrome. J Am Heart Assoc 2016;5:1.

34 Chen B, Frangogiannis NG. Immune cells in repair of the infarcted myocardium. Microcirculation 2017;24:e12305.

35 Schillinger M, Exner M, Mlekusch W, et al. Balloon angioplasty and stent implantation induce a vascular inflammatory reaction. $J$ Endovasc Ther 2002;9:59-66.

36 Clinicaltrials.gov. CLEAR SYNERGY Neutrophil Substudy - Full Text View, 2021. Available: https://clinicaltrials.gov/ct2/show/ NCT03874338 [Accessed 6 June 2021]. 